\title{
Development of an analytical method for dibutyl phthalate (DBP) determination in water samples using gas chromatography
}

\author{
Sabina Ziembowicz ${ }^{1, *}$, Matgorzata Kida $^{1}$, and Piotr Koszelnik ${ }^{1}$ \\ ${ }^{1}$ Department of Environmental Engineering and Chemistry, Faculty of Civil and Environmental \\ Engineering and Architecture, Rzeszów University of Technology, Powstanców Warszawy 6, \\ 35-959 Rzeszów, Poland
}

\begin{abstract}
The aim of the work described here was to develop and validate a method by which dibutyl phthalate may be subject to determinations using solid phase extraction and gas chromatography. Optimization of the chromatographic method was based on the selection of working conditions for both the chromatograph and the detector. Following the optimization of extraction and separation parameters, the method was validated by evaluating specificity, the analytical curve, linearity, limits of detection and quantification and recovery. The proposed method has been evaluated in terms of linearity, over a range of concentrations from 0 to $7.5 \mathrm{mg} \cdot \mathrm{L}^{-1}$. The analytical curves show values for correlation coefficients higher than 0.99 . Mean recoveries from samples ranged from 97 to $127 \%$, with relative standard deviation lower than $11 \%$. Limit of detection LOD and limit of quantification LOQ values were 0.02 and $0.053 \mathrm{mg} \cdot \mathrm{L}^{-1}$ respectively.
\end{abstract}

\section{Introduction}

In recent years, there has been increasing concern over the environmental risks posed by the so-called emerging contaminants (ECs). ECs originate from a variety of product types, including pharmaceuticals, nanomaterials, personal-care products, paints and coatings. Some ECs, such as natural products and transformation products of synthetic chemicals, may be formed in the environment by biochemical processes in animals, plants and microbes [1-6]. Among the ECs cited most frequently it is phthalates that are of particular concern, due to their ubiquity and presence in the environment at higher levels generally than in the case of other ECs [7]. The esters of phthalic acid (PAEs) are used in a wide variety of products, such as enteric coatings of pharmaceutical pills and nutritional supplements, building materials, personal-care products, medical devices, detergents, children's toys, modelling clay, waxes, printing inks and textiles. They are applied widely to improve the flexibility of polyvinyl chloride (PVC). However, they are not bound to the plastic covalently, and thus leak into the environment [8]. For example, the concentrations of dimethyl phthalate (DMP) in atmospheric particulate matter, fresh water and sediments,

\footnotetext{
*Corresponding author: s.ksiazek@prz.edu.pl
} 
soil, and landfills are N.D. $-10.4 \mathrm{ng} \cdot \mathrm{m}^{-3}$, N.D. $-31.7 \mu \mathrm{g} \cdot \mathrm{L}^{-1}$, N.D. $-316 \mu \mathrm{g} \cdot \mathrm{kg}^{-1}$ dry weight and N.D. $-200 \mu \mathrm{g} \cdot \mathrm{kg}^{-1}$ dry weight, N.D. $-43.27 \mu \mathrm{g} \cdot \mathrm{L}^{-1}$, respectively [9].

Because of their extensive and widespread application, phthalates are ubiquitous in all parts of the environment. Some PAEs (e.g. DEHP - di(2-ethylhexyl) phthalate and DBP - dibutyl phthalate) and their metabolites are suspected endocrine-disrupting chemicals that also exhibit carcinogenic action [10]. It is further suspected that the phthalate esters play a role in male reproductive health problems, and they have also been associated with several other problems, including allergy and asthma [11]. Dibutyl phthalate is one representative of the phthalate acid esters that has found applications in a wide variety of industrial and common household products. In addition, it is used in the manufacture of adhesives, dyes, lacquers, personal-care products, cosmetics, and more. The human population may be exposed to dibutyl phthalate by inhalation, ingestion or dermal contact at the workplace, through the use of consumer products, and indirectly via the environment. Human exposure via the environment may occur through contact with contaminated air, water, soil or food $[12,13]$. Fig. 1 shows the chemical structure of DBP, while its physicochemical properties are as presented in Table 1.

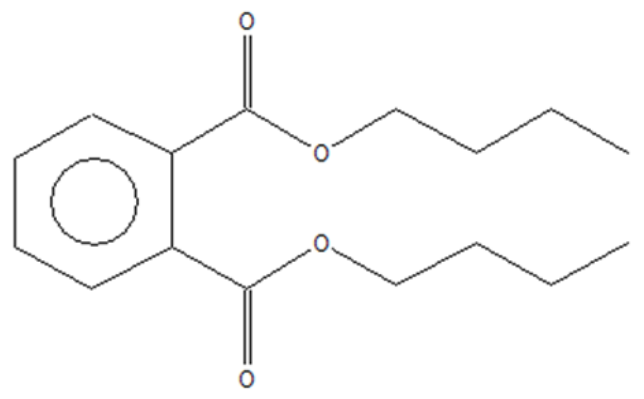

Fig. 1. Chemical structure of DBP.

Table 1. Physicochemical properties of DBP $[14,15]$.

\begin{tabular}{|c|c|}
\hline Identification & Information \\
\hline Chemical Name & 1,2 -Benzenedicarboxylic acid, dibutyl ester \\
\hline CAS Number & $84-74-2$ \\
\hline Chemical Formula & $\mathrm{C}_{16} \mathrm{H}_{22} \mathrm{O}_{4}$ \\
\hline Molecular Weight & 278.34 \\
\hline Physical State & Oily liquid \\
\hline Color & Colorless to faint yellow \\
\hline Odor & Slight ester-like \\
\hline Melting Point & $-35^{\circ} \mathrm{C}$ \\
\hline Boiling Point & $340^{\circ} \mathrm{C}$ \\
\hline Vapor Pressure & $2.7 \times 10^{-5} \mathrm{~mm} \mathrm{Hg}$ \\
\hline Solubility & Water: $11.2 \mathrm{mg} \cdot \mathrm{L}^{-1}$ Organic solvents: very soluble \\
\hline Log Kow & 4.45 \\
\hline Flashpoint & $157^{\circ} \mathrm{C}$ \\
\hline Incompatibilities & Liquid chlorine \\
\hline
\end{tabular}

The aim of the work detailed here was to develop and validate a method by which to make determinations for dibutyl phthalate in water samples, using solid phase extraction and gas chromatography. 


\section{Experimental}

\subsection{Chemicals and materials}

All chemicals used were of analytical reagent grade. Standard $(1 \mathrm{mg} / \mathrm{ml})$ solutions of dibutyl phthalate and benzyl benzoate (BB) were obtained from Sigma Aldrich (Saint Louis, USA). Ultra-pure water was obtained from Purix CNX-100, while $3 \mathrm{~mL}$ SPE cartridges and C18 sorbents were purchased from SiliCycle (Quebec City, Canada). Analytical grade dichloromethane, methanol, and acetone were also purchased from POCH (Gliwice, Poland).

\subsection{Preparation of standard solutions}

Individual stock solutions of dibutyl phthalate and benzyl benzoate $\left(1 \mathrm{mg} \cdot \mathrm{mL}^{-1}\right)$ were prepared in methanol and stored at $4^{\circ} \mathrm{C}$. Calibration standard solutions containing $1,5,10$, 50,100 and $150 \mu \mathrm{g} / \mathrm{mL}$ of DBP with $10 \mu \mathrm{g} / \mathrm{mL}$ of BB were prepared daily in methanol. Determinations were performed based on the peak area of analyte to internal standard ratio.

\subsection{Glassware and reagent control}

To avoid PAE contamination, all glassware used in the study was washed, dried, soaked in acetone and dried at $280^{\circ} \mathrm{C}$ for at least $5 \mathrm{~h}$. All the glassware and reagents were checked for potential phthalate contamination.

\subsection{Sample preparation: solid phase extraction (SPE)}

Prior to extraction, the SPE columns were conditioned with $5 \mathrm{ml}$ methanol under a vacuum (Fig. 2), following addition of $5 \mathrm{~mL}$ deionized water. The $20 \mathrm{ml}$ deionized water was spiked with standard solutions of dibutyl phthalate $\left(0-7.5 \mathrm{mg} \cdot \mathrm{L}^{-1}\right)$ and with the internal standard BB $(10 \mu \mathrm{g})$, and subsequently passed through the column at a flow rate of $1 \mathrm{~mL} \cdot \mathrm{min}^{-1}$. The dibutyl phthalate was eluted with methanol and dichloromethane and the collected fraction concentrated down to $1 \mathrm{~mL}$ under nitrogen. The percentage recovery was carried out using gas chromatography.

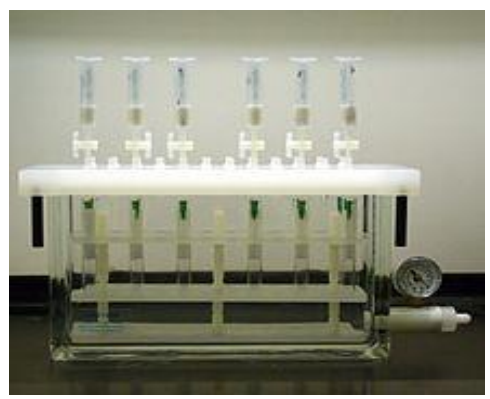

Fig. 2. SPE extraction system. 


\subsection{Chromatography analysis}

Chromatography analysis was performed using a GC-2010 Plus system (Shimadzu) with an FID detector, LabSolutions software and AOC-20i Autosampler. The GC experimental conditions were as shown in Table 2.

Table 2. Chromatography analysis parameters.

\begin{tabular}{|c|c|}
\hline Carrier gas & Hydrogen, air, helium \\
\hline Column & ZB-5MSplus, $30 \mathrm{~m} \times 0.25 \mathrm{~mm} \times 0.25 \mu \mathrm{m}$ \\
\hline Oven program & $60^{\circ} \mathrm{C}(1 \mathrm{~min})-20^{\circ} \mathrm{C} / \mathrm{min}$ to $300^{\circ} \mathrm{C}(5 \mathrm{~min})$ \\
\hline Injector temperature & $280^{\circ} \mathrm{C}$ \\
\hline Detector temperature & $250^{\circ} \mathrm{C}$ \\
\hline Injection volume & $1 \mu \mathrm{L}$ \\
\hline
\end{tabular}

\subsection{Method validation}

Method validation entailed the evaluation of specificity, linearity, repeatability, recovery, limit of detection (LOD) and limit of quantification (LOQ). Quantification was performed using the internal calibration method based on a seven-point calibration curve. Benzyl benzoate was used as the internal standard for the quantification of DBP.

\section{Results and discussion}

The work described here attempted to develop the GC method for the determination of concentrations of dibutyl phthalate in water. Figure 3 shows a typical chromatogram for DBP. Retention times were found to be $11.88 \pm 0.1$ minutes in the case of DBP, and $10.96 \pm 0.1$ minutes for $\mathrm{BB}$. The chromatogram was obtained under the GC conditions referred to in Table 2. It was concluded that the method presented allowed for the satisfactory isolation of DBP from other matrix substances. The specificity of the method was determined using a blank solution $(\mathrm{MeOH})$, a solution spiked with DBP, a solution spiked with the internal standard (BB) and a solution with DBP and BB. Spiking the sample with analyte did not cause peak splitting, with retentions times staying the same as with the corresponding peaks from test solutions. The chromatogram for blank solution revealed no peaks with retention times of DBP and BB.

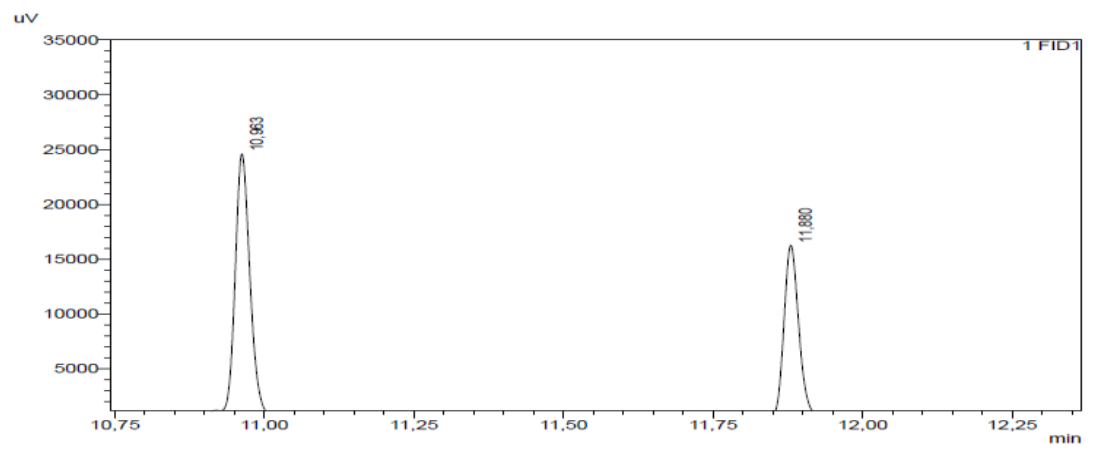

Fig. 3. Typical chromatogram of DBP (10.96 min) and BB (11.88 min). 
The linearity of the analytical method was checked by preparing and injecting six standard solutions. For each concentration level three replicates were made prepared using the stock standard solution. Following the injection and chromatographic run of each standard solution, the calibration curve of the peak area versus concentration was evaluated (Fig. 4). The value of the coefficient for the correlation was 0.9999 . The method was thus proven to obtain chromatographic signals directly proportional to the concentration of the analytes over the entire range investigated.

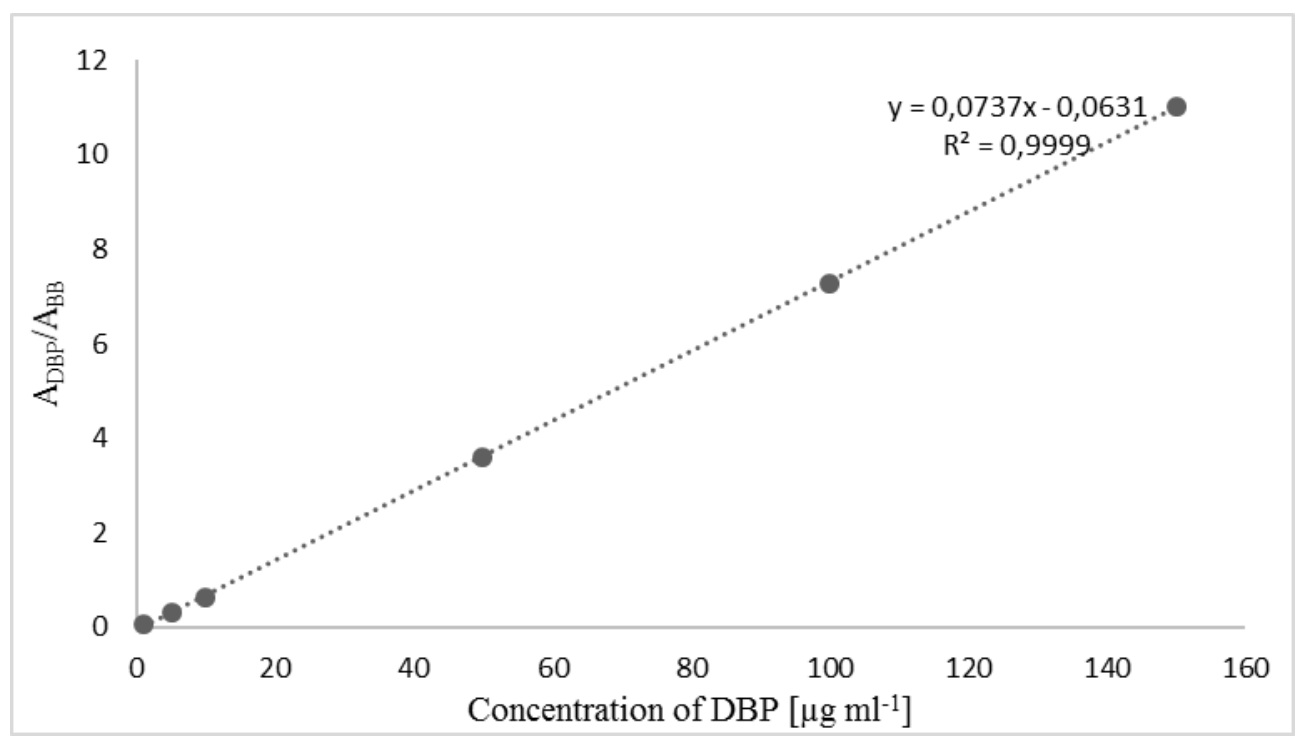

Fig. 4. Calibration curve for DBP.

Accuracy was calculated by reference to the recovery percentage for the analysis. To prove the method's validity, DBP recovery was estimated by analyzing a water sample spiked with $0.05,0.25,0.5,2.5,5$ and $7.5 \mathrm{mg} \cdot \mathrm{L}^{-1}$ of DBP. Across these levels, the efficiency of the SPE method was satisfactory. The results from the optimization of the extraction procedure are shown in Table 3. Recoveries for DPB resulted in values in the range $97-127 \%$.

Table 3. Recovery results for DBP.

\begin{tabular}{|c|c|c|c|c|c|c|}
\hline Concentration of DBP $\left[\mathbf{m g} \cdot \mathbf{L}^{-1}\right]$ & $\mathbf{0 . 0 5}$ & $\mathbf{0 . 2 5}$ & $\mathbf{0 . 5}$ & $\mathbf{2 . 5}$ & $\mathbf{5}$ & $\mathbf{7 . 5}$ \\
\hline Mean recovery $[\%]$ & 124 & 127 & 120 & 111 & 98 & 97 \\
\hline
\end{tabular}

Precision can be expressed in terms of relative standard deviation (RSD). This was assessed using six different spiked concentrations and six replicates for each concentration. RSD values in the $2.54-10.56 \%$ range were achieved for the spiked concentrations referred to above. 
Table 4. Precision of the method.

\begin{tabular}{|c|c|c|c|c|c|c|}
\hline \multirow{2}{*}{$\mathbf{n}=\mathbf{6}$} & \multicolumn{5}{|c|}{ Concentration of DBP $\left[\mathbf{m g} \cdot \mathbf{L}^{-1}\right]$} \\
\cline { 2 - 7 } & $\mathbf{0 . 0 5}$ & $\mathbf{0 . 2 5}$ & $\mathbf{0 . 5}$ & $\mathbf{2 . 5}$ & $\mathbf{5}$ & $\mathbf{7 . 5}$ \\
\hline RSD [\%] & 10.56 & 3.12 & 3.66 & 4.14 & 2.54 & 3.86 \\
\hline
\end{tabular}

The LOD and LOQ values were found to be 0.02 and $0.053 \mathrm{mg} \cdot \mathrm{L}^{-1}$ respectively, making the level of sensitivity of the equipment used clear. The limit of detection (LOD) was calculated as the concentration generating a peak about 3 times as high as the noise height, while the limit of quantification (LOQ) was calculated as the concentration generating a peak about 10 times as high as the noise height [16].

\section{Conclusion}

$\checkmark$ An analytical method for the determination of DBP in water using SPE and GC-FID analysis has been developed and validated.

$\checkmark$ Parameter linearity, recovery, precision, detection limits and quantification limits were all shown to be acceptable.

$\checkmark$ A six-point calibration curve was constructed over the concentration range of interest $\left(1-150 \mu \mathrm{g} \cdot \mathrm{mL}^{-1}\right)$, using external standards. Calibration curve regression coefficients $\left(\mathrm{R}^{2}\right)$ assumed values above 0.99 .

$\checkmark \quad$ The method (SPE extraction) presented an average recovery of $97 \%$ and $127 \%$.

$\checkmark$ Repeatability was measured using relative standard deviations (RSD) at all concentration levels, and was found to range from $2.54 \%$ to $10.56 \%$.

$\checkmark$ Obtained LOD and LOQ values were of 0.02 and $0.053 \mathrm{mg} \cdot \mathrm{L}^{-1}$ respectively.

\section{References}

1. I. Rykowska, W. Wasiak, Open Chem. 13, 1, 1353-1370 (2015)

2. J. Kaleta, M. Kida, P. Koszelnik, D. Papciak, A. Puszkarewicz, B. TchórzewskaCieślak, Arch. Environ. Prot. 43, 3, 32-41 (2017)

3. K. Pochwat, D. Słyś, S. Kordana, J. Hydrol. 549, 501-511 (2017)

4. M. Smol, M. Włodarczyk-Makuła, K. Mielczarek, J. Bohdziewicz, D. Włóka, Polycycl. Aromat Comp. 36, 20-39 (2016)

5. A. Rosińska, L. Dąbrowska, Desalin. Water Treat. 51, (7-9), 1657-1663 (2013)

6. J. Naumczyk, P. Marcinowski, J. Bogacki, Environ. Prot. Eng. 43, 2 (2017)

7. K. Manamsa, E. Crane, M. Stuart, J. Talbot, D. Lapworth, A. Hart, Sci. Total Environ. 568, 712-726 (2016)

8. J. Huang, P.N. Nkrumah, Y. Li, G. Appiah-Sefah, Rev. Environ. Contam. T, Springer New York 224, 39-52 (2013)

9. D.W. Gao, Z.D. Wen, Sci. Total Environ. 541, 986-1001 (2016)

10. F. Zeng, K. Cui, Z. Xie, L. Wu, M. Liu, G. Sun, Z, Zeng, Environ. Pollut. 156, 2, 425-434 (2008) 
11. Q. Zeng, C. Wei, Y. Wu, K. Li, S. Ding, J. Yuan, M. Chen, Food Chem. Toxicol. 56, 18-27 (2013)

12. E. Mylchreest, M. Sar, R.C. Cattley, P.M. Foster, Toxicol. Appl. Pharm. 156, 2, 81-95 (1999)

13. A.K. Müller, E. Nielsen, O. Ladefoged, The Danish Veterinary and Food Administration (2003)

14. Agency for Toxic Substances and Disease Registry (ATSDR). Toxicological Profile for Di-n-butyl Phthalate. US Department of Health and Human Services (2001)

15. National Industrial Chemicals Notification and Assessment Scheme (NICNAS). Existing Chemical Hazard Assessment Report Dibutyl Phthalate (2008)

16. A. Shrivastava, V.B. Gupta, Chron. Young Sci. 2, 1, 21 (2011) 